\title{
Rhythmic behavior in a two-population mean-field Ising model
}

\author{
Francesca Collet \\ Institute of Applied Mathematics, Delft University of Technology, Mekelweg 4, 2628 CD Delft, The Netherlands
}

Marco Formentin ${ }^{\dagger}$ and Daniele Tovazzi ${ }^{\ddagger}$

Dipartimento di Matematica, Università degli Studi di Padova, Via Trieste 63, 35121 Padova, Italy

(Received 22 June 2016; revised manuscript received 30 August 2016; published 28 October 2016)

\begin{abstract}
Many real systems composed of a large number of interacting components, as, for instance, neural networks, may exhibit collective periodic behavior even though single components have no natural tendency to behave periodically. Macroscopic oscillations are indeed one of the most common self-organized behavior observed in living systems. In the present paper we study some dynamical features of a two-population generalization of the mean-field Ising model with the scope of investigating simple mechanisms capable to generate rhythms in large groups of interacting individuals. We show that the system may undergo a transition from a disordered phase, where the magnetization of each population fluctuates closely around zero, to a phase in which they both display a macroscopic regular rhythm. In particular, there exists a region in the parameter space where having two groups of spins with inter- and intrapopulation interactions of different strengths suffices for the emergence of a robust periodic behavior.
\end{abstract}

DOI: 10.1103/PhysRevE.94.042139

\section{INTRODUCTION}

Living systems are characterized by the emergence of recurrent dynamical patterns at all scales of magnitude. Selforganized collective behaviors are observed both in large communities of microscopic components, like neural oscillations and gene network activity, as well as on larger levels, such as predator-prey equilibria, applauding audiences, and flocks of birds. In particular, collective periodic behaviors are among the most commonly observed ways of self-organization in biology, ecology, and socioeconomics [1-4]. The attempt of modeling such complex systems leads naturally to considering large families of microscopic identical units. Complexity and self-organization then arise on a macroscopic scale from the dynamics of these minimal components that evolve coupled by interaction terms.

Within this scenario, we are interested in particle systems whose macroscopic observables oscillate between different ordered phases. In these models, microscopic units neither have a tendency to behave periodically on their own-in contrast to Kuramoto rotators [5] —nor are subject to a periodic forcing. Nevertheless, the particles organize to produce a very regular motion perceived only macroscopically: a collective self-sustained rhythm. Various stylized models have been proposed to capture the essence of this phenomenon, but in most of them rigorous results are hard to obtain, as the study ends up looking for stable attractors of nonlinear infinite dimensional dynamical systems [6,7]. Analytically tractable models can be obtained by considering mean-field interactions. Recently, the existence of periodic collective behaviors has been proven for some classes of mean-field systems derived as perturbation of classical reversible ferromagnetic models by adding a dissipation term [8-10]. Dissipation dumps the

\footnotetext{
*f.collet-1@tudelft.nl

${ }^{\dagger}$ formen@math.unipd.it

†tovazzi@math.unipd.it
}

influence of interaction when no transition occurs for a long time. The simplest spin system within this class is the dissipative mean-field Ising model proposed in Refs. $[9,10]$. Coupled diffusions with dissipation have been considered in Ref. [8]. Besides dissipation, delay in the interactions may also produce rhythmic behavior in mean-field systems as highlighted in Ref. [11] for interacting Hawkes processes and in Ref. [12] for spin-glass models. Indeed, theoretical models based on mean-field interacting spin systems, although simplistic, are able to show a good qualitative description of cooperative macroscopic behavior in self-organizing systems. In the past few decades, for this reason and their analytical tractability, they have also been applied in social sciences [13-15], finance [16,17], chemistry [18], and ecology [19,20].

An interesting family, which has naturally emerged in applications, is a multispecies extension of the mean-field Ising model. The possibility of taking into account several kinds of magnetic spins is a peculiar feature that may be relevant to capture diverse phenomena from magnetism in anisotropic materials to social issues. A two-population version of the Curie-Weiss model was introduced in the 1950s to mimic the phase transition undergone by metamagnets [21]. Recently, it has been receiving renewed attention due to its ability to describe the large-scale behavior of socioeconomic systems, such as cultural coexistence, immigration, and integration [15,22-24]. Multipopulated noninteracting spin models are the cornerstone of McFadden discrete choice theory [25]. The extension of the discrete choice theory to the interacting, and more realistic case has been done in Ref. [15] and represents an important step toward the understanding of collective behaviors in societies. From an equilibrium viewpoint, the investigation of the two-species model introduced in Ref. [15] has been pursued at a mathematical level in Ref. [26], where the thermodynamic limit has been rigorously obtained. In the present paper, following the work started in Ref. [27], we continue the analysis of the dynamical features of this two-population generalization of the mean-field Ising spin system. 
Inspired by previous works with applications in biology [28], neurosciences [11], and socioeconomics [12], our purpose is to investigate mechanisms that enhance rhythmic behavior. The goal of the paper is not to have a comprehensive study of the dynamics but rather to show the onset of regular behavior. Our main finding indicates that having two groups of spins with possibly different sizes and different interand intrapopulation interactions suffices for the emergence of macroscopic oscillations. Additional mechanisms as dissipated or delayed interactions are not necessary. However, delay may produce periodic behavior in interaction network configurations where otherwise absent. In our approach, the transition to rhythm is detected in the thermodynamic limit via the presence of a Hopf bifurcation. Stable limit cycles may also emerge from nonlocal bifurcations [8], but there is no numerical evidence it could be the case for the class of mean-field systems considered here.

\section{MODEL}

The two-population Curie-Weiss model is a spin system where on the complete graph two types of spins are present. Particles are differentiated by their mutual interactions: There are two intragroup interactions, tuning how strongly sites in the same group feel each other, and two intergroup interactions, giving the magnitude of the influence between particles of distinct populations. Let $S=\{-1,+1\}$ be the state space of a single spin variable and let $\sigma=\left(\sigma_{j}\right)_{j=1}^{N} \in S^{N}$ be the $N$-site configuration. We divide the whole system of size $N$ into two disjoint subsystems of sizes $N_{1}$ and $N_{2}$, respectively. Let $I_{1}$ (respectively, $I_{2}$ ) be the set of sites belonging to the first (respectively, second) subsystem. We have $\operatorname{card}\left(I_{1}\right)=$ $N_{1}$ and $\operatorname{card}\left(I_{2}\right)=N_{2}$, with $N_{1}+N_{2}=N$. To fix notation, let $1,2, \ldots, N_{1}$ be the indices corresponding to particles in population $I_{1}$ and $N_{1}+1, N_{1}+2, \ldots, N$ those of particles in population $I_{2}$, so

$$
\sigma=\left(\begin{array}{c|c}
\text { Population } I_{1} & \text { Population } I_{2} \\
\left(\sigma_{1}, \sigma_{2}, \ldots, \sigma_{N_{1}}\right. & \left.\sigma_{N_{1}+1}, \sigma_{N_{1}+2}, \ldots, \sigma_{N}\right)
\end{array}\right.
$$

Given two spins, their mutual interaction depends on the subsystems to which they belong. In our setting, $J_{11}$ and $J_{22}$ tune the interaction within sites of the same subsystem, whereas $J_{12}$ and $J_{21}$ control the coupling strength between spins located in different subsystems (see Fig. 1 for a schematic representation). All the interactions can be either positive or negative, allowing both ferromagnetic and antiferromagnetic interactions.

We want to define two different Markovian dynamics in this setting. Let us denote by

$$
m_{N_{i}}(t):=\frac{1}{N_{i}} \sum_{j \in I_{i}} \sigma_{j}(t)
$$

the magnetization of population $I_{i}(i=1,2)$ at time $t$. Moreover, if $\alpha:=N_{1} / N$ is the proportion of sites belonging to the first group, we introduce the functions

$$
\begin{aligned}
& R_{1}\left(x_{1}, x_{2}\right)=\alpha J_{11} x_{1}+(1-\alpha) J_{12} x_{2}, \\
& R_{2}\left(x_{1}, x_{2}\right)=(1-\alpha) J_{22} x_{2}+\alpha J_{21} x_{1} .
\end{aligned}
$$

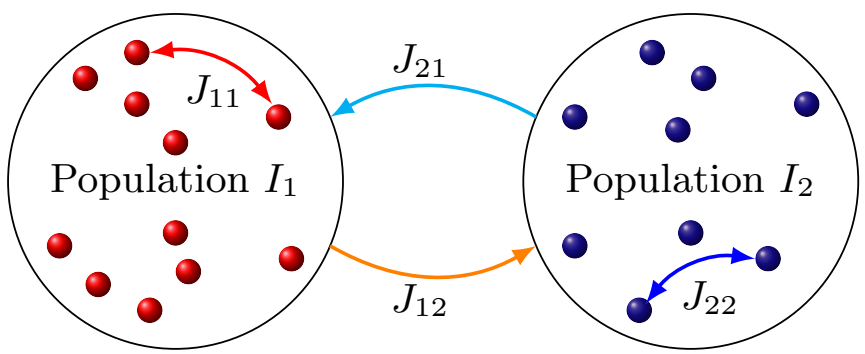

FIG. 1. A schematic representation of the interaction network for a bipartite Curie-Weiss model. Spins are divided into two populations $I_{1}$ and $I_{2}$. Within $I_{1}$ (respectively, $I_{2}$ ) particles feel a mean-field interaction with coupling $J_{11}$ (respectively, $J_{22}$ ). In addition, population $I_{1}$ (respectively, $I_{2}$ ) influences the dynamics of the other group through its magnetization with strength $J_{12}$ (respectively, $J_{21}$ ).

The $R_{i}$ 's are composed of two terms: The first one tells us how strongly sites in the same population interact, while the second encodes the way one population influences the other.

We are now ready to describe the two dynamics we are interested in. For reasons that will be clear in a moment, throughout the paper we will refer to these dynamics as "without delay" and "with delay."

\section{A. Microscopic dynamics without delay}

Let $\sigma^{i}$ denote the configuration obtained from $\sigma$ by flipping the $i$ th spin. At any time $t$ the system may experience a transition whose rate depends on the magnetization vector at time tonly.

The transition $\sigma \longrightarrow \sigma^{i}$ occurs at rate

$$
\begin{array}{ll}
e^{-\sigma_{i} R_{1}\left(m_{N_{1}}(t), m_{N_{2}}(t)\right)}, & \text { if } i \in I_{1} \\
e^{-\sigma_{i} R_{2}\left(m_{N_{1}}(t), m_{N_{2}}(t)\right)}, & \text { if } i \in I_{2} .
\end{array}
$$

These are standard Glauber Markovian dynamics where, for any small $\delta>0$, the transition probability $P\{\sigma(t+$ $\delta) \mid \sigma(s), s \leqslant t\}$ depends only on the configuration at time $t$, i.e., $P\{\sigma(t+\delta) \mid \sigma(s), s \leqslant t\}=P\{\sigma(t+\delta) \mid \sigma(t)\}$.

\section{B. Microscopic dynamics with delay}

A second type of dynamics in which a delay kernel acts on the intergroup interactions is also of interest. At any time $t$ the influence of each population on the other is given by an average over the magnetization trajectory up to time $t$, weighted through a delay kernel.

The transition $\sigma \longrightarrow \sigma^{i}$ occurs at rate

$$
\begin{array}{ll}
e^{-\sigma_{i} R_{1}\left(m_{N_{1}}(t), \gamma_{N_{2}}^{(n)}(t)\right)}, & \text { if } i \in I_{1} \\
e^{-\sigma_{i} R_{2}\left(\gamma_{N_{1}}^{(n)}(t), m_{N_{2}}(t)\right)}, & \text { if } i \in I_{2},
\end{array}
$$

where, for $n \in \mathbb{N}$ and $k \in \mathbb{N} \backslash\{0\}$, we define

$$
\gamma_{N_{i}}^{(n)}(t)=\int_{0}^{t} \frac{(t-s)^{n}}{n !} k^{n+1} e^{-k(t-s)} m_{N_{i}}(s) d s,
$$

for $i=1,2$. The delay kernel is in the form of Erlang distribution. The parameter $n$ is related to the shape of the bump of the function; whereas $k$ tunes how sharp and close to time $t$ the peak is. In particular, for any fixed $n \in \mathbb{N}$ and large $k$, the kernel has a sharp peak around $s \simeq t$, where the 
maximum is attained. As a consequence, in the limit as $k$ goes to infinity, only values of the magnetizations close to $m_{N_{i}}(t)$, for $i=1,2$, enter the dynamics and the rates (D) approach the rates (wD). This indicates that the addition of delay is relevant for small values of the parameter $k$.

\section{RESULTS}

We want to characterize the infinite volume limits of the dynamics (wD) and (D) described above. The strategy is to determine a suitable Markov process whose dynamics can be derived from the original microscopic dynamics and then apply standard techniques of convergence of generators to get weak convergence to the thermodynamic limiting evolution [29, Corollary 8.7, Chapter 4]. This machinery has been applied in detail in Ref. [19] for the treatment of a similar system.

Note that the limit as $N$ goes to infinity must be taken in such a way the proportions $\alpha$ and $1-\alpha$ of the two groups remain constant. In the sequel, we will write $m_{i}(\cdot)$ for the infinite volume limit of $m_{N_{i}}(\cdot)$. Analogously, $\gamma_{i}^{(n)}(\cdot)$ will be the limit of $\gamma_{N_{i}}^{(n)}(\cdot)$.

\section{A. Macroscopic dynamics without delay}

The dynamics (wD) for configurations induce a Markovian evolution on the magnetization vector $\left(m_{N_{1}}(t), m_{N_{2}}(t)\right)$. As $N \longrightarrow \infty$, the process $\left(m_{N_{1}}(t), m_{N_{2}}(t)\right)_{t \geqslant 0}$ weakly converges to the solution of the system of ordinary differential equations,

$$
\begin{aligned}
\dot{m}_{1}(t)= & 2 \sinh \left[R_{1}\left(m_{1}(t), m_{2}(t)\right)\right] \\
& -2 m_{1}(t) \cosh \left[R_{1}\left(m_{1}(t), m_{2}(t)\right)\right] \\
\dot{m}_{2}(t)= & 2 \sinh \left[R_{2}\left(m_{1}(t), m_{2}(t)\right)\right] \\
& -2 m_{2}(t) \cosh \left[R_{2}\left(m_{1}(t), m_{2}(t)\right)\right] .
\end{aligned}
$$

\section{B. Macroscopic dynamics with delay}

In this case, the magnetization vector in itself does not inherit Markovianity from (D). To get a Markovian evolution for macroscopic observables, we have to consider the process

$$
\left(m_{N_{1}}(t), m_{N_{2}}(t),\left(\gamma_{N_{1}}^{(j)}(t)\right)_{j=0}^{n},\left(\gamma_{N_{2}}^{(j)}(t)\right)_{j=0}^{n}\right)_{t \geqslant 0},
$$

that, as $N \longrightarrow \infty$, weakly converges to the solution of the following system of ordinary differential equations,

$$
\begin{aligned}
& \dot{m}_{1}(t)= 2 \sinh \left[R_{1}\left(m_{1}(t), \gamma_{2}^{(n)}(t)\right)\right] \\
&-2 m_{1}(t) \cosh \left[R_{1}\left(m_{1}(t), \gamma_{2}^{(n)}(t)\right)\right] \\
& \dot{m}_{2}(t)=2 \sinh \left[R_{2}\left(\gamma_{1}^{(n)}(t), m_{2}(t)\right)\right]-2 m_{2}(t) \cosh \left[R_{2}\left(\gamma_{1}^{(n)}(t), m_{2}(t)\right)\right] \\
& \dot{\gamma}_{1}^{(0)}(t)=k\left[-\gamma_{1}^{(0)}(t)+m_{1}(t)\right] \\
& \dot{\gamma}_{1}^{(n)}(t)=k\left[-\gamma_{1}^{(n)}(t)+\gamma_{1}^{(n-1)}(t)\right], \text { for } n>0 \\
& \dot{\gamma}_{2}^{(0)}(t)=k\left[-\gamma_{2}^{(0)}(t)+m_{2}(t)\right] \\
& \dot{\gamma}_{2}^{(n)}(t)=k\left[-\gamma_{2}^{(n)}(t)+\gamma_{2}^{(n-1)}(t)\right], \text { for } n>0 .
\end{aligned}
$$

We remark that introducing delay through a kernel (an idea borrowed from Ref. [11]) leads to a finite-dimensional macroscopic dynamics. In contrast, if instead of $\gamma_{N_{i}}^{(n)}(t)$ we choose $\bar{\gamma}_{N_{i}}=m_{N_{i}}(t-\tau)$, with fixed $\tau>0$ (delayed rates), the limiting dynamics are infinite dimensional. A detailed analysis of a mean-field spin system with delayed rates is given in Ref. [12]. In addition therein, the author considers a spatial model where both the interaction and the delay depend on respective locations of sites.

It is evident from equation (MD) that, for large $k$, there is a separation of time scales between the evolutions of the $m$ variables and the $\gamma$ variables: The latter relax to their equilibrium point much faster than the former. By applying the center manifold reduction [30, Theorem 5.2], it is possible to reduce the $(2 n+4)$-dimensional dynamical system (MD) to a planar dynamical system, describing the $O(1)$ evolution of $\left(m_{1}, m_{2}\right)$ on the center manifold,

$$
\gamma_{1}^{(0)}=\cdots=\gamma_{1}^{(n)}=m_{1} \text { and } \gamma_{2}^{(0)}=\cdots=\gamma_{2}^{(n)}=m_{2} .
$$

Thus we can neglect the equations for the $\gamma$ variables and consider only the dynamics of the $m$ variables after having substituted the stationary values for the $\gamma$ 's. It follows that, for large $k$, the reduction of (MD) coincides with the macroscopic dynamics without delay (MwD). This calls for a few observations:

(i) The proximity between the macroscopic evolutions (MwD) and (MD) we obtain for large $k$ is not so unexpected. It somehow reflects the fact that the microscopic dynamics (wD) and (D) are close to each other as $k$ goes to infinity. Indeed, since $\gamma_{N_{i}}^{(n)}(t) \stackrel{k \rightarrow+\infty}{\longrightarrow} m_{N_{i}}(t)$, for all $n \in \mathbb{N}$ and $t \in \mathbb{R}^{+}$, there is convergence of the transition rates (D) towards (wD) and, as a consequence, the two stochastic processes become close to each other.

(ii) The proximity between the dynamics (MwD) and (MD) holds true only in the large $k$ limit. It will be clear in the next section that, for small $k$, the qualitative behaviors of the two systems may significantly differ. Delay may produce periodic behavior in interaction network configurations where otherwise absent.

\section{Transition from disorder to rhythm}

We want to detect the transition from a disordered behavior, where $m_{N_{1}}(\cdot)$ and $m_{N_{2}}(\cdot)$ fluctuate around zero, to a collective rhythmic behavior in which we have periodic motion of the magnetizations (see Figs. 2 and 3). To this aim, we consider the limiting evolutions (MwD) and (MD) and we look for the presence of a Hopf bifurcation. Recall that a (supercritial) Hopf bifurcation occurs when a stable periodic orbit arises from an equilibrium point as, at some critical values of the parameters, it loses stability. Such a bifurcation can be detected when a pair of complex eigenvalues of the linearized system around the equilibrium crosses the imaginary axis [31, Theorem 2, Chapter 4.4].

We start by considering the set of ordinary differential equations given by (MwD). It is immediate to verify that the origin is an equilibrium for all values of the parameters. 

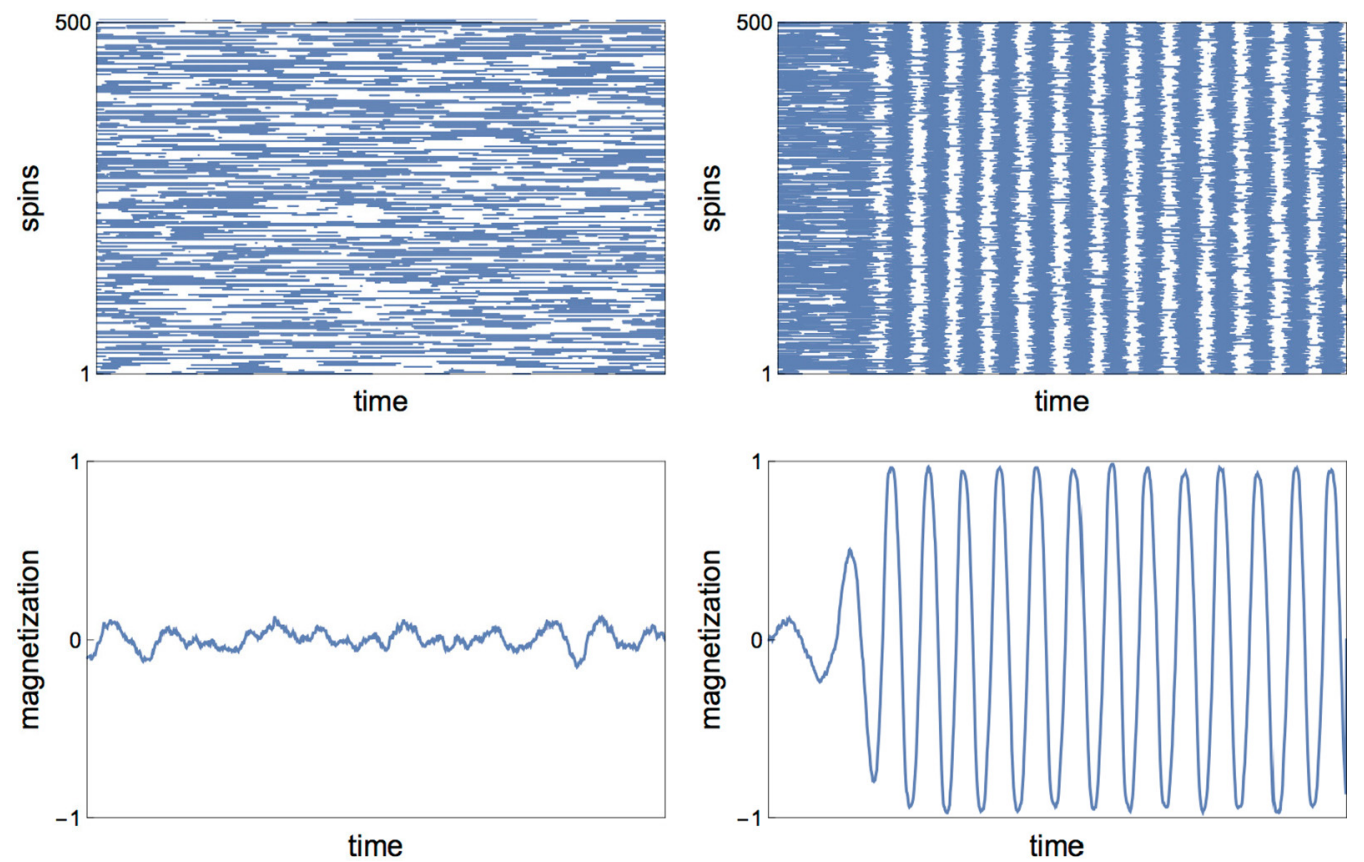

FIG. 2. Transition from disordered behavior (on the left) to collective rhythm (on the right) for the spin system (wD). Simulations have been run with $N=1000, \alpha=1 / 2, J_{12}=-6, J_{21}=5$, and $J_{11}=J_{22}=0.5$ on the left and $J_{11}=J_{22}=3$ on the right. The top row shows the time evolution of all the spins belonging to population $I_{1}$. Spins are labeled from 1 to 500 on the $y$ axis. Blue spots represent +1 spins, whereas white spots stand for -1 . In the bottom line the corresponding evolution for the magnetization is depicted.

Therefore we analyze the spectrum of the linearization of the dynamics $(\mathrm{MwD})$ around $(0,0)$ to understand if there exist parameter values for which the origin loses stability whenever a pair of pure imaginary conjugate eigenvalues appear. The characteristic polynomial of the linearized system reads

$$
\begin{aligned}
P(\lambda)= & \lambda^{2}-\lambda\left[2 \alpha J_{11}+2(1-\alpha) J_{22}-4\right] \\
& +4\left(\alpha J_{11}-1\right)\left[(1-\alpha) J_{22}-1\right] \\
& -4 \alpha(1-\alpha) J_{12} J_{21} .
\end{aligned}
$$

It follows that a Hopf bifurcation occurs if and only if both the conditions

$$
\begin{aligned}
& \alpha J_{11}=2-(1-\alpha) J_{22} \\
& \left((1-\alpha) J_{22}-1\right)^{2}+\alpha(1-\alpha) J_{12} J_{21}<0
\end{aligned}
$$

are satisfied. In particular, we have:

(a) If $J_{11}, J_{22} \leqslant 0$, the equality in (3) is never satisfied and thus system $(\mathrm{MwD})$ never undergoes a Hopf bifurcation.

(b) If $J_{12} J_{21} \geqslant 0$, the inequality in (3) has no solution and then again it is impossible to find a Hopf bifurcation.

(c) In the set $\left\{J_{11}, J_{22} \leqslant 0\right\}^{c} \cap\left\{J_{12} J_{21} \geqslant 0\right\}^{c}$ we can choose properly the values of the parameters to get a Hopf bifurcation.

Our aim is now to understand if, in those regions of the parameter space where (MwD) does not undergo a Hopf bifurcation, we may produce a transition to periodic motion by adding delay in the dynamics. In this respect we move to the analysis of (MD). It is easy to see that whenever a Hopf bifurcation is present for (MwD), the same holds also for (MD). Delay may of course change the critical value at which the bifurcation occurs but not its presence. Moreover, by adding delay we can induce rhythmicity in a subspace of the phase $J_{11}, J_{22} \leqslant 0$, where periodic orbits were absent for (MwD).

We consider the set of mean-field equations in (MD) with $J_{11}, J_{22}<0$ and $J_{12} J_{21}<0$. We then linearize the dynamics around the null $(2 n+4)$-dimensional vector, which indeed is fixed point of (MD) for all parameter values, and we study the spectrum. We remark that, since dealing with a nonplanar dynamical system, to detect a supercritical Hopf bifurcation it does not suffice looking for a pair of pure imaginary conjugate eigenvalues; in addition, it is necessary to check that all the $2 n+2$ remainings have negative real part.

To simplify computations assume

$$
2\left(\alpha J_{11}-1\right)=-k \quad \text { and } \quad 2\left[(1-\alpha) J_{22}-1\right]=-k,
$$

so

$$
\alpha J_{11}=(1-\alpha) J_{22}=-\frac{k}{2}+1,
$$

with $k>2$. The constant $k$ we are using here is the same appearing in the definition of the delay kernel. Let us denote by $x_{j}$, with $j=0,1, \ldots, 2 n+3$, the $j$ th eigenvalue of the linearization of (MD) around the null solution. For $j=$ $0,1, \ldots, 2 n+3$, we get

$$
x_{j}=-k-|A|^{\frac{1}{2 n+4}} k^{\frac{n+1}{n+2}} \exp \left\{i \frac{(2 j+1) \pi}{2 n+4}\right\},
$$

where $A=4 \alpha(1-\alpha) J_{12} J_{21}<0$. Therefore, for

$$
k=|A|^{\frac{1}{2}}\left[\cos \left(\frac{\pi}{2 n+4}\right)\right]^{n+2}
$$



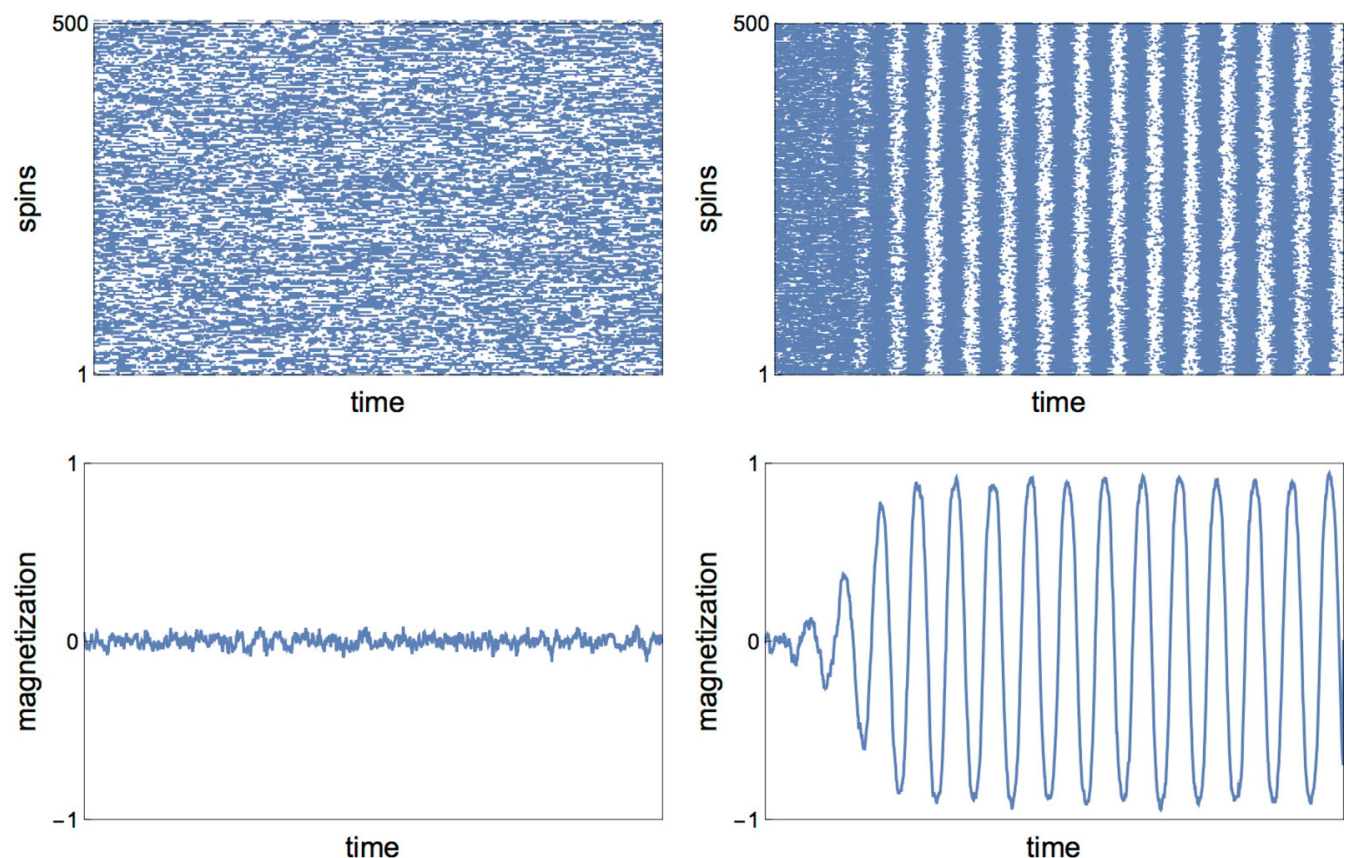

FIG. 3. Transition from disordered behavior (on the left) to collective rhythm (on the right) for the spin system (D) when both the intragroup interactions are negative (i.e., $J_{11}, J_{22}<0$ ). Simulations have been run with $N=1000, \alpha=1 / 2, n=2, J_{12}=5, J_{21}=-6$, and $J_{11}=J_{22}=-4$ with $k=6$ on the left and $J_{11}=J_{22}=-1$ with $k=3$ on the right. Recall that the value of $k$ depends on $J_{11}$ and $J_{22}$ through assumption (4). The top row shows the time evolution of all the spins belonging to population $I_{1}$. Spins are labeled from 1 to 500 on the $y$ axis. Blue spots represent +1 spins; whereas white spots stand for -1 . In the bottom line the corresponding evolution for the magnetization is depicted.

TABLE I. Qualitative summary of the results. In the left column a schematic representation of the considered interaction network is displayed. The color convention for couplings is as in Fig. 1. For each interaction network we highlight the possibility of observing or not observing periodic behavior when considering the dynamics (MwD) (central column) or (MD) (right column). Notice that, in all cases except for one, delay is not necessary to produce rhythmic oscillations.

\begin{tabular}{lll}
\hline & Dynamics & \\
\hline Interactions without delay delay & with \\
\hline & $\begin{array}{c}\text { Rhythmic } \\
\text { behavior }\end{array}$ & $\begin{array}{c}\text { Rhythmic } \\
\text { behavior }\end{array}$
\end{tabular}

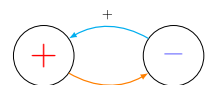

Rhythmic

Rhythmic behavior behavior

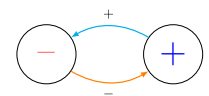

Rhythmic behavior

Rhythmic behavior

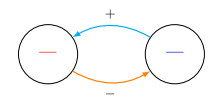

Rhythrinc behavior a Hopf bifurcation occurs, as $x_{n+1}$ and $x_{n+2}$ are the two first eigenvalues passing the imaginary axis with positive derivative. See Fig. 3.

We qualitatively summarize our findings in Table I. The table gives information about the possible emergence of macroscopic oscillations for the interaction network configurations depicted in the left column. Given a type of interaction network, the corresponding dynamical systems (MwD) (central column) and (MD) (right column) may or may not exhibit rhythmic behavior. When writing they do, we mean that there exists a choice of the parameters [satisfying (3) for (MwD) and (5) for (MD)] for which a Hopf bifurcation occurs at the origin. We observe that the parameter $n$ does not influence the presence (absence) of periodic behavior but simply modifies the threshold value for the phase transition. Referring to the table, notice that only when both the intragroup interactions are negative (i.e., $J_{11}, J_{22}<0$ ), delay is needed to enhance the transition to a periodic behavior for $\left(m_{1}, m_{2}\right)$. In all other cases a robust choice of the parameters is sufficient. In particular, delay is not necessary to create a limit cycle when $J_{11}, J_{12}<0$ and $J_{22}, J_{21}>0$ (third row in the table). The particle system constructed on the latter interaction network resembles one of the models introduced in Ref. [12], where, however, a fixed time delay is present in the rates of transition. Our results indicate that it is not delay but rather the asymmetry of the coupling strengths that is the crucial feature to produce a collective rhythm. We believe that for the mean-field spin glass in Ref. [12] the introduction of delay becomes necessary due to the fine choice of the interactions: $\left|J_{11}\right|=\left|J_{22}\right|=\left|J_{12}\right|=\left|J_{21}\right|=J$, with $J>0$. 


\section{CONCLUSIONS}

In the present paper we investigated the emergence of collective periodic behavior in a two-population generalization of the mean-field Ising model. We analyzed the role of interaction network and delay in enhancing an oscillatory evolution for the magnetization vector. We were interested in showing that it is possible to induce a transition from a disordered phase, where $m_{N_{1}}$ and $m_{N_{2}}$ fluctuate closely around zero, to a phase in which they both display a macroscopic regular rhythm. In particular, we have proven that a robust choice of the coupling constants and of the population sizes is sufficient for a limit cycle to arise. Moreover, in the case when the choice of the parameters does not suffice to favor the transition, delay may help in this respect (see Table I).

When considering the dynamics without delay, the mechanism behind the emergence of periodicity can be understood in the following terms. If the intrapopulation interaction strengths $J_{11}$ and $J_{22}$ are large enough, each single population can be seen as a macrospin that under Glauber dynamics tends to its own rest state. However, as soon as the two populations are linked together within an interaction network with $J_{12} J_{21}<0$, they form a frustrated pair of macrospins where the rest state of the first is not compatible with the rest position of the second. As a consequence, the dynamics is not driven to a fixed equilibrium and continues oscillating. This intuition suggests that the creation of a collective rhythm by splitting the particles in two groups differentiated by their mutual interactions is very much related to the mean-field setting, a conclusion that is supported also by numerics. We ran simulations of a two-population version of the nearest-neighbor Ising model on a finite square lattice of side length $N \gg 1$ (total number of spins of order $10^{3}$ ).

(i) Particles are randomly divided in two distinct groups: Each site in the box is assigned to population $I_{1}$ (respectively, $I_{2}$ ) with probability $\alpha$ (respectively, $1-\alpha$ ), with $\left.\alpha \in\right] 0,1[$.

(ii) At any time $t$, the spin $\sigma_{k}$ flips at a rate of the form (wD) in which the mean-field magnetizations $m_{N_{i}}(t)(i=1,2)$ are replaced by local magnetizations

$$
\ell_{i}(h, t)=\sum_{\substack{j \sim h \\ j \in I_{i}}} \sigma_{j}(t) \quad(i=1,2),
$$

where the sum is extended only to sites $j$ nearest neighbors of $h$ (as the symbol $\sim$ is intended to mean).

(iii) Periodic boundary conditions are considered.

With diverse simulations we explored the parameter space and did not find any oscillatory evolution for the global magnetizations of the two groups. The reason is that in this setting the short-range of interaction destroys the macrospin structure of each family of spins and does not allow for the creation of a frustrated macronetwork. The addition of delay does not change the scenario.

To conclude, recall that the results we obtained for the two-population Curie-Weiss model are derived in the limit as the number of particles goes to infinity and the passage from an incoherent to a coherent behavior of the magnetization vector is detected by the occurrence of a (supercritical) Hopf bifurcation. It is worth mentioning that the presence of a stable limit cycle as attractor for the dynamics is a pure infinite volume effect. The finite $N$ system (wD) is an irreducible, time-homogeneous Markov process and therefore it relaxes to a time-independent invariant distribution. As a consequence, periodicity turns out to be only a metastable state for the finite-size system.

\section{ACKNOWLEDGMENTS}

We thank Pierluigi Contucci and Paolo Dai Pra for useful discussions, comments, and suggestions. F.C. was supported by the Netherlands Organisation for Scientific Research (NWO) via TOP-1 Grant No. 613.001.552. M.F. acknowledges the University of Padova, Mathematics Department Senior Grant No. 795/2014 Prot. 59008 for financial support.
[1] W.-C. Chen, Nonlinear dynamics and chaos in a fractional-order financial system, Chaos Soliton. Fract. 36, 1305 (2008).

[2] G. B. Ermentrout and D. H. Terman, Mathematical Foundations of Neuroscience, Vol. 35 (Springer, Berlin, 2010).

[3] W. Weidlich and G. Haag, Concepts and Models of a Quantitative Sociology: The Dynamics of Interacting Populations, Vol. 14 (Springer, Berlin, 2012).

[4] P. Turchin and A. D. Taylor, Complex dynamics in ecological time series, Ecology 73, 289 (1992).

[5] Y. Kuramoto, Self-entrainment of a population of coupled nonlinear oscillators, in International Symposium on Mathematical Problems in Theoretical Physics (Kyoto Univ., Kyoto, 1975), Lecture Notes in Physics, Vol. 39. (Springer, Berlin, 1975), pp. 420-422.

[6] G. Giacomin and C. Poquet, Noise, interaction, nonlinear dynamics and the origin of rhythmic behaviors, Braz. J. Probab. Stat. 29, 460 (2015).
[7] B. Lindner, J. Garcia-Ojalvo, A. Neiman, and L. SchimanskyGeier, Effects of noise in excitable systems, Phys. Rep. 392, 321 (2004).

[8] F. Collet, P. Dai Pra, and M. Formentin, Collective periodicity in mean-field models of cooperative behavior, NoDEA 22, 1461 (2015).

[9] P. Dai Pra, M. Fischer, and D. Regoli, A Curie-Weiss model with dissipation, J. Stat. Phys. 152, 37 (2013).

[10] P. Dai Pra, G. Giacomin, and D. Regoli, Noise-induced periodicity: Some stochastic models for complex biological systems, in Mathematical Models and Methods for Planet Earth (Springer, Berlin, 2014), pp. 25-35.

[11] S. Ditlevsen and E. Löcherbach, Multi-class oscillating systems of interacting neurons, arXiv:1512.00265 [Stoch. Proc. Appl. (to be published)].

[12] J. Touboul, The hipster effect: When anticonformists all look the same, arXiv:1410.8001. 
[13] W. A. Brock and S. N. Durlauf, Discrete choice with social interactions, Rev. Econom. Stud. 68, 235 (2001).

[14] F. Collet, P. Dai Pra, and E. Sartori, A simple mean field model for social interactions: dynamics, fluctuations, criticality, J. Stat. Phys. 139, 820 (2010).

[15] P. Contucci and S. Ghirlanda, Modeling society with statistical mechanics: An application to cultural contact and immigration, Qual. Quant. 41, 569 (2007).

[16] P. Dai Pra, W. J. Runggaldier, E. Sartori, and M. Tolotti, Large portfolio losses; a dynamic contagion model, Ann. Appl. Probab. 19, 347 (2009).

[17] R. Frey and J. Backhaus, Pricing and hedging of portfolio credit derivatives with interacting default intensities, Int. J. Theor. Appl. Finance 11, 611 (2008).

[18] A. Di Biasio, E. Agliari, A. Barra, and R. Burioni, Mean-field cooperativity in chemical kinetics, Theor. Chem. Acc. 131, 1 (2012).

[19] C. Borile, P. Dai Pra, M. Fischer, M. Formentin, and A. Maritan, Time to absorption for a heterogeneous neutral competition model, J. Stat. Phys. 156, 119 (2014).

[20] I. Volkov, J. R. Banavar, S. P. Hubbell, and A. Maritan, Neutral theory and relative species abundance in ecology, Nature 424, 1035 (2003).

[21] P. Schiffer and I. Daruka, Two-population model for anomalous low-temperature magnetism in geometrically frustrated magnets, Phys. Rev. B 56, 13712 (1997).
[22] A. Barra, P. Contucci, R. Sandell, and C. Vernia, An analysis of a large dataset on immigrant integration in Spain. The statistical mechanics perspective on social action, Sci. Rep. 4, 1 (2014).

[23] I. Gallo, A. Barra, and P. Contucci, Parameter evaluation of a simple mean-field model of social interaction, Math. Models Methods Appl. Sci. 19, 1427 (2009).

[24] P. Contucci, I. Gallo, and G. Menconi, Phase transitions in social sciences: Two-population mean field theory, Int. J. Mod. Phys. B 22, 2199 (2008).

[25] D. McFadden, Economic choices, Am. Econ. Rev. 91, 351 (2001).

[26] I. Gallo and P. Contucci, Bipartite mean field spin systems. Existence and solution, Math. Phys. Electron. J. 14, 1 (2008).

[27] F. Collet, Macroscopic limit of a bipartite Curie-Weiss model: A dynamical approach, J. Stat. Phys. 157, 1301 (2014).

[28] R. Fernández, L. R. Fontes, and E. J. Neves, Density-profile processes describing biological signaling networks: Almost sure convergence to deterministic trajectories, J. Stat. Phys. 136, 875 (2009).

[29] S. N. Ethier and T. G. Kurtz, Markov Processes: Characterization and Convergence (John Wiley \& Sons, New York, 1986).

[30] Y. A. Kuznetsov, Elements of applied bifurcation theory, in Applied Mathematical Sciences, Vol. 112, 3rd ed. (SpringerVerlag, New York, 2004).

[31] L. Perko, Differential Equations and Dynamical Systems (Springer-Verlag, New York, 2001). 